\title{
Invariants of a Pair of Conics Revisited
}

Long QUAN

\author{
Patrick GROS
}

LIFIA - IMAG, 46, avenue Felix Viallet, 38031 Grenoble, France
Roger MOHR

Abstract

The invariants of a pair of quadratic forms and a pair of coplanar conics are revisited following $[1,5,2]$. For a given pair of conics, with their associated matrices $\mathbf{C}_{1}$ and $\mathbf{C}_{2}$, we show that $\operatorname{Trace}\left(\mathbf{C}_{2}^{-1} \mathbf{C}_{1}\right), \operatorname{Trace}\left(\mathbf{C}_{1}^{-1} \mathbf{C}_{2}\right)$ and $\left|\mathbf{C}_{1}\right| /\left|\mathbf{C}_{2}\right|$ are only invariants of associated quadratic forms, but not invariants of the conics. Two of true invariants of the conics are $\frac{\text { Trace }\left(\mathbf{C}_{2}^{-1} \mathbf{C}_{1}\right)}{\left(\text { Trace }\left(\mathbf{C}_{1}^{-1} \mathbf{C}_{2}\right)\right)^{2}} \frac{\left|\mathbf{C}_{2}\right|}{\left|\mathbf{C}_{1}\right|}$ and $\frac{\text { Trace }\left(\mathbf{C}_{1}^{-1} \mathbf{C}_{2}\right)}{\left(\text { Trace }\left(\mathbf{C}_{2}^{-1} \mathbf{C}_{1}\right)\right)^{2}} \frac{\left|\mathbf{C}_{1}\right|}{\left|\mathbf{C}_{2}\right|}$. Then the true invariants of the conics are geometrically interpreted, in terms of cross ratios, through the common self-polar triangle of the two conics.

\section{Introduction}

Following a series of publications of Forsyth et al. [1, 6, 2], invariants of a pair of coplanar conics provoke much interest. We first review some general aspects of invariants of a pair of conics, with reference to Semple and Kneebone [5]. We emphasize the difference between invariants of quadratics and that of conics. For a given pair of conics, with their associated matrices $\mathbf{C}_{1}$ and $\mathbf{C}_{2}$, we show that Trace $\left(\mathbf{C}_{2}^{-1} \mathbf{C}_{1}\right)$, Trace $\left(\mathbf{C}_{1}^{-1} \mathbf{C}_{2}\right)$ and $\left|\mathbf{C}_{1}\right| /\left|\mathbf{C}_{2}\right|$ are only invariants of associated quadratic forms, but not invariants of the conics. Two of true invariants of the conics are $\frac{\operatorname{Trace}\left(\mathbf{C}_{2}^{-1} \mathbf{C}_{1}\right)}{\left(\operatorname{Trace}\left(\mathbf{C}_{1}^{-1} \mathbf{C}_{2}\right)\right)^{2}} \frac{\left|\mathbf{C}_{2}\right|}{\left|\mathbf{C}_{1}\right|}$ and $\frac{\operatorname{Trace}\left(\mathbf{C}_{2}^{-1} \mathbf{C}_{2}\right)}{\left(\operatorname{Trace}\left(\mathbf{C}_{2}^{-1} \mathbf{C}_{1}\right)\right)^{2}} \frac{\left|\mathbf{C}_{1}\right|}{\left|\mathbf{C}_{2}\right|}$. Secondly, geometric interpretation of conic invariants based on cross ratios is presented using the common self-polar triangle. Note that in [3], Kapur and Mundy have tried to make a geometrical interpretation for their proposed invariants, but it is not complete. The results presented in this paper can be considered as a complementary part of the work by Forsyth et al. [1, 2], Zisserman et al. [6] and Kapur et al. [3], in order to better understand conic invariants and to make better pratical use of them.

The general aspect on the theory of invariants is developed by Semple and Kneebone [5] in chapter 8. Most of demonstrations of fundamental projective properties used in this paper are not explicited, the readers can find them in [5], particularly in chapter 5 dealing with conics. So, for more detailed discussion on algebraic projective geometry, interested peoples are invited to read [5]. 


\section{A brief review of the theory of invariants}

\subsection{Invariants of a pair of quadratic forms}

Consider a quadratic form: $\mathcal{Q}=x^{t} \mathbf{C} x=\sum_{i, j} a_{i j} x_{i} x_{j}, i, j=1,2,3$; where $\mathbf{C}$ is a symmetric $3 \times 3$ matrix and $x=\left(x_{1}, x_{2}, x_{3}\right)^{t}$ is the vector of homogeneous coordinates in the plane. We suppose $|\mathbf{C}| \neq 0$, where $|\mathbf{C}|$ is the discriminant of $\mathbf{C}$; thus insures the associated conics are not degenerate. We change the coordinates by the substitution $\boldsymbol{x}=\mathbf{P} \boldsymbol{x}^{\prime}, \mathbf{P}$ can be any non-singular transformation $(|\mathbf{P}| \neq 0)$. We look for invariants which are independent of any $\mathbf{P}$.

It follows $\mathbf{C}^{\prime}=\mathbf{P}^{t} \mathbf{C P}$. The associated discriminants have the important property $\left|\mathbf{C}^{\prime}\right|=|\mathbf{P}|^{2}|\mathbf{C}|$.

Let $\mathcal{Q}_{1}=x^{t} \mathbf{C}_{1} x$ and $\mathcal{Q}_{2}=x^{t} \mathbf{C}_{2} x$ be two quadratic forms in $x=\left(x_{1}, x_{2}, x_{3}\right)^{t}$. The general linear combination of $\mathcal{Q}_{1}$ and $\mathcal{Q}_{2}$ may be written as $\mathcal{Q}_{\lambda, \mu}=$ $\lambda \mathcal{Q}_{1}+\mu \mathcal{Q}_{2}=x^{t} \mathbf{C}_{\lambda, \mu} x$.

Therefore, $\left|\mathbf{C}_{\lambda, \mu}\right|=I_{1} \lambda^{3}+I_{2} \lambda^{2} \mu+I_{3} \lambda \mu^{2}+I_{4} \mu^{3}$, where,

$$
\begin{aligned}
3 I_{1} & =a_{11} A_{11}+a_{22} A_{22}+a_{33} A_{33}+2 a_{12} A_{12}+2 a_{23} A_{23}+2 a_{13} A_{13}=3\left|\mathbf{C}_{1}\right|, \\
I_{2} & =b_{11} A_{11}+b_{22} A_{22}+b_{33} A_{33}+2 b_{12} A_{12}+2 b_{23} A_{23}+2 b_{13} A_{13}, \\
I_{3} & =a_{11} B_{11}+a_{22} B_{22}+a_{33} B_{33}+2 a_{12} B_{12}+2 a_{23} B_{23}+2 a_{13} B_{13}, \\
3 I_{4} & =b_{11} B_{11}+b_{22} B_{22}+b_{33} B_{33}+2 b_{12} B_{12}+2 b_{23} B_{23}+2 b_{13} B_{13}=3\left|\mathbf{C}_{2}\right| ;
\end{aligned}
$$

$a_{i j}$ and $b_{i j}$ being elements of $\mathbf{C}_{1}$ and $\mathbf{C}_{2} ; A_{i j}$ and $B_{i j}$ the cofactors of $a_{i j}$ and $b_{i j}$.

Take any non-singular linear transformation $\mathbf{P}, \mathcal{Q}_{1}, \mathcal{Q}_{2}$ and $\mathcal{Q}_{\lambda, \mu}$ are respectively transformed by $x=\mathbf{P} x^{\prime}$ into the forms

$$
\mathcal{Q}_{1}^{\prime}=x^{\prime t} \mathbf{C}_{1}^{\prime} x^{\prime}, \mathcal{Q}_{2}^{\prime}=x^{\prime t} \mathbf{C}_{2}^{\prime} x^{\prime} \text { and } \mathcal{Q}_{\lambda, \mu}^{\prime}=\lambda \mathcal{Q}_{1}^{\prime}+\mu \mathcal{Q}_{2}^{\prime}=x^{\prime t} \mathbf{C}_{\lambda, \mu}^{\prime} x^{\prime}
$$

Write $\left|\mathbf{C}_{\lambda, \mu}^{\prime}\right|=I_{1}^{\prime} \lambda^{3}+I_{2}^{\prime} \lambda^{2} \mu+I_{3}^{\prime} \lambda \mu^{2}+I_{4}^{\prime} \mu^{3}$, since $\left|\mathbf{C}_{\lambda, \mu}^{\prime}\right|=|\mathbf{P}|{ }^{2}\left|\mathbf{C}_{\lambda, \mu}\right|$, which holds for any $\lambda$ and $\mu$, we obtain

$$
I_{1}^{\prime}=|\mathbf{P}|^{2} I_{1}, \quad I_{2}^{\prime}=|\mathbf{P}|^{2} I_{2}, \quad I_{3}^{\prime}=|\mathbf{P}|^{2} I_{3}, \quad \text { and } I_{4}^{\prime}=|\mathbf{P}|^{2} I_{4} .
$$

As a consequence, any ratios of $I_{i} / I_{j}$, for $i \neq j$ are independent of any transformation $\mathbf{P}$, and thus are invariants for the discussed pair of quadratic forms.

\subsection{Invariants of a pair of conics}

Let consider the conics associated with these quadratic forms. It should be noted that the conics are associated in such a way, not with uniquely defined quadratic forms $\mathcal{Q}_{1}$ and $\mathcal{Q}_{2}$, but with families of forms $\lambda \mathcal{Q}_{1}$ and $\mu \mathcal{Q}_{2}$, where $\lambda$ and $\mu$ are arbitrary scalar. The ratios $I_{i} / I_{j}$ are no more invariant for the associated conics, since invariants of conics have to be also independent of any multiplicative scalar $\lambda$ and $\mu$ for their matrices. Therefore, the weights of $I_{i}$, $i=1,2,3,4$; with respect to $\lambda$ and $\mu$, have to be taken into account. Two invariants for a pair of conics can be obtained (cf. [5]) by

$$
\alpha=\frac{I_{1} I_{3}}{I_{2}^{2}} \quad \text { and } \quad \beta=\frac{I_{2} I_{4}}{I_{3}^{2}}
$$


which are independent not only of any transformation $\mathbf{P}$ but also of any $\lambda$ and $\mu$. In addition, any other invariant which can be derived in this way is a rational function of $\alpha$ and $\beta$. It is evident that all invariants of conics are those of associated quadratic forms.

\section{Geometric interpretation of invariants of a pair of conics}

\subsection{Some basic concepts}

The cross ratio of four points $P_{i}, i=1,2,3,4$, with their homogenous coordinates $\left(\lambda_{i}, \mu_{i}\right)$ on a projective line is defined by

$$
\left\{P_{1}, P_{2} ; P_{3}, P_{4}\right\}=\frac{\left(\theta_{1}-\theta_{3}\right) /\left(\theta_{2}-\theta_{3}\right)}{\left(\theta_{1}-\theta_{4}\right) /\left(\theta_{2}-\theta_{4}\right)} \text {, with } \theta_{i}=\frac{\mu_{i}}{\lambda_{i}} \text {. }
$$

By convention, $\left\{P_{1}, P_{2} ; P_{3}, \infty\right\}=\left(\theta_{1}-\theta_{3}\right) /\left(\theta_{2}-\theta_{3}\right)$.

For a given conic $\mathcal{C}$, if $Q$ and $R$ are two points of the plane, $Q$ and $R$ are said to be conjugate points with respect to the conic $\mathcal{C}$ if they are harmonic with respect to the points $P_{1}, P_{2}$ which are the intersection points of $Q R$ on the conic (cf. Figure 1), i.e. $\left\{Q, R ; P_{1}, P_{2}\right\}=-1$.

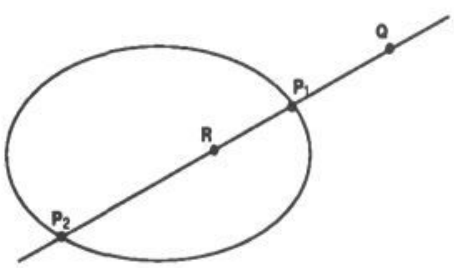

Figure 1: $Q$ and $R$ are conjugate points with respect to the conic.

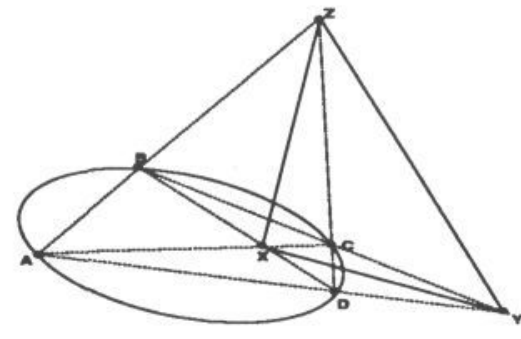

Figure 2: A self-polar triangle $X Y Z$ for a conic determined by four points of the conic $A, B, C, D$.

The locus of points conjugated to a fixed point $P$ is a line. This line is called the polar of $P$. For a given line $l$, there is a unique point which is conjugate to every points of $l$ with respect to $\mathcal{C}$. This point is called the pole of $l$. The conic $\mathcal{C}$ thus defines a transformation of the points (poles) of the plane into the lines (polars) of the plane and lines (polars) into points (poles). Recall that lines and points are dual entities and have the same (formal) homogeneous coordinates representation in projective plane. In matrix notation, this transformation (known as the polarity defined by $\mathcal{C}$ ) can be written as $\lambda l_{\text {polar }}=\mathrm{C} P$ and $\mu P_{\text {pole }}=\mathrm{Cl}$; where $\lambda$ and $\mu$ are any non-zero scalars.

If the polar of $P$ passes through a point $Q$, then the polar of $Q$ passes through $P$.

The three points $Q_{1}, Q_{2}, Q_{3}$ are said to form a self-polar triangle for $\mathcal{C}$, if the polar of each is the line joining the other two. There is an infinity of self-polar triangles for a given conic. 


\subsection{Where is the common self-polar triangle?}

If $A, B, C, D$ are four points of the conic $\mathcal{C}$, the diagonal triangle $X Y Z$ of the quadrangle $A B C D$ is self-polar for $\mathcal{C}$ (cf. Figure 2).

Consider two coplanar conics $\mathcal{C}_{1}$ and $\mathcal{C}_{2}$, they always intersect in four points, say $A, B, C, D$. $A, B, C, D$ form a quadrangle whose diagonal triangle $X, Y, Z$ is self-polar both with respect to $\mathcal{C}_{1}$ and $\mathcal{C}_{2}$. That is, it is the common self-polar triangle of $\mathcal{C}_{1}$ and $\mathcal{C}_{2}$ (cf. Figure 3 ). This common self-polar triangle is unique.

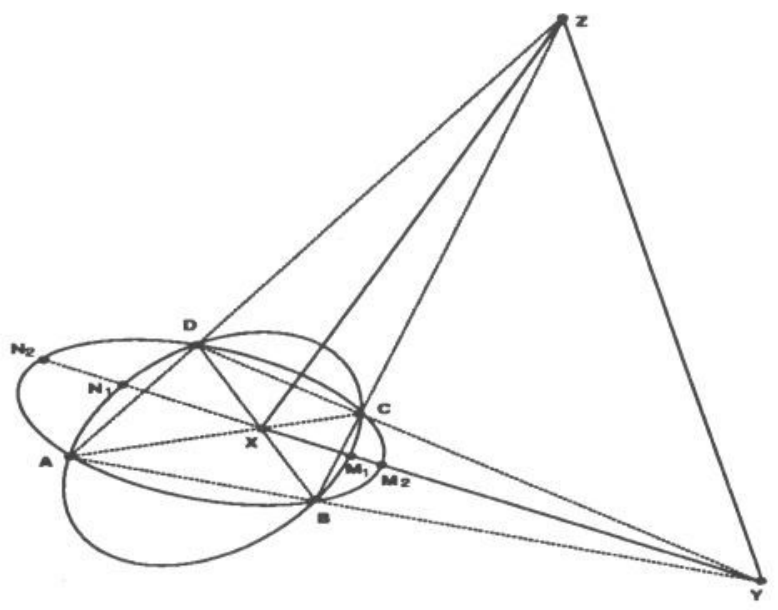

Figure 3: The common self-polar triangle $X Y Z$ for a pair of coplanar conics.

The common self-polar triangle of two conics is the diagonal triangle of the quadrangle defined by the intersection points of the two conics.

\subsection{What are the vertices of the common self-polar tri- angle for?}

The polar of $X$ with respect to $\mathcal{C}_{1}$ can be obtained by $\mu l_{X}=\mathrm{C}_{1} X$ and the polar of $X$ with respect to $\mathcal{C}_{2}$ by $\nu l_{X}^{\prime}=\mathrm{C}_{2} X$. As $X Y Z$ is the common self-polar triangle, $l_{X}$ and $l_{X}^{\prime}$ are the same polar $Y Z$ of $X$. Homogeneous coordinates for points and lines are determined apart from an arbitrary scalar factor. Two lines are the same if $l_{X}=\lambda l_{X}^{\prime}$. Therefore $\mathbf{C}_{1} X=\lambda \mathbf{C}_{2} X$, leading to $\left(\mathbf{C}_{2}^{-1} \mathbf{C}_{1}\right) X=$ $\lambda X$. This is the standard definition for eigenvalue and eigenvector, meaning $X$ is the eigenvector of $\mathbf{C}_{2}^{-1} \mathbf{C}_{1}$. Similarly, we can prove that the other two vertices $Y$ and $Z$ are also eigenvectors of $\mathbf{C}_{2}^{-1} \mathbf{C}_{1}$.

The three vertices of the common self-polar triangle are the three eigenvectors of $\mathbf{C}_{2}^{-1} \mathbf{C}_{1}$.

\subsection{What are the eigenvalues of $\mathrm{C}_{2}^{-1} \mathrm{C}_{1}$ related to?}

Since the three vertices of the common self-polar triangle are the three eigenvectors of $\mathbf{C}_{2}^{-1} \mathbf{C}_{1}$, so what are the eigenvalues of $\mathbf{C}_{2}^{-1} \mathbf{C}_{1}$ related to? 
Let now see how the equation of a conic may be put in a simple form by taking a special triangle of reference.

When the triangle of reference is self-polar, the equation of a conic reduces to the diagonal form: $a_{11} x_{1}^{2}+a_{22} x_{2}^{2}+a_{33} x_{3}^{2}=0$.

When the common self-polar triangle $X Y Z$ is selected as the triangle of reference, i.e. $X=(1,0,0)^{t}, Y=(0,1,0)^{t}$ and $Z=(0,0,1)^{t}, \mathcal{C}_{1}$ and $\mathcal{C}_{2}$ reduce at the same time to the diagonal form. These diagonal forms are obtained by the substitution $x=\mathbf{P} x^{\prime}$, where $\mathbf{P}=\left(X^{t} Y^{t} Z^{t}\right)$.

$$
\begin{aligned}
& \mathcal{C}_{1}^{\prime}: x^{\prime t} \mathrm{C}_{1} x^{\prime}=a_{11}^{\prime} x_{1}^{\prime 2}+a_{22}^{\prime} x_{2}^{\prime 2}+a_{33}^{\prime} x_{3}^{\prime 2}=0 \\
& \mathcal{C}_{2}^{\prime}: x^{\prime t} \mathrm{C}_{2} x^{\prime}=b_{11}^{\prime} x_{1}^{\prime 2}+b_{22}^{\prime} x_{2}^{\prime 2}+b_{33}^{\prime} x_{3}^{\prime 2}=0 .
\end{aligned}
$$

The equations of the two conics can be written as:

$$
\begin{aligned}
x^{t} \mathbf{C}_{1} x & =\left(\mathbf{P} x^{\prime}\right)^{t} \mathbf{C}_{1}\left(\mathbf{P} x^{\prime}\right)=x^{\prime t}\left(\mathbf{P}^{t} \mathbf{C}_{1} \mathbf{P}\right) x \\
x^{t} \mathbf{C}_{2} x & =\left(\mathbf{P} x^{\prime}\right)^{t} \mathbf{C}_{2}\left(\mathbf{P} x^{\prime}\right)=x^{\prime t}\left(\mathbf{P}^{t} \mathbf{C}_{2} \mathbf{P}\right) x
\end{aligned}
$$

Thus, $\mathbf{C}_{2}^{\prime-1} \mathbf{C}_{1}^{\prime}=\left(\mathbf{P}^{t} \mathbf{C}_{2} \mathbf{P}\right)^{-1}\left(\mathbf{P}^{t} \mathbf{C}_{1} \mathbf{P}\right)=\mathbf{P}^{-1}\left(\mathbf{C}_{2}^{-1} \mathbf{C}_{1}\right) \mathbf{P}$.

Recall that the matrix $\mathbf{P}$ is formed by the eigenvectors $X, Y, Z$ of $\mathbf{C}_{2}^{-1} \mathbf{C}_{1}$, so we prove that $\mathbf{C}_{2}^{-1} \mathbf{C}_{1}$ and $\mathbf{C}_{2}^{\prime-1} \mathbf{C}_{1}^{\prime}$ have the same eigenvalues, noted as $\lambda_{1}, \lambda_{2}$ and $\lambda_{3}$. They can be directly obtained by

$$
\lambda_{1}=\frac{a_{11}^{\prime}}{b_{11}^{\prime}}, \quad \lambda_{2}=\frac{a_{22}^{\prime}}{b_{22}^{\prime}} \text { and } \lambda_{3}=\frac{a_{33}^{\prime}}{b_{33}^{\prime}} \text {. }
$$

The polar $X Y$ meets each of two conics in two points. $M_{1}, N_{1}$ for $\mathcal{C}_{1}$ and $M_{2}, N_{2}$ for $\mathcal{C}_{2}$. The equation of $X Y$ reduces to $x_{3}^{\prime}=0$. These intersection points can be obtained by solving the following equations.

$$
\left\{\begin{array} { l } 
{ a _ { 1 1 } ^ { \prime } x _ { 1 } ^ { \prime 2 } + a _ { 2 2 } ^ { \prime } x _ { 2 } ^ { \prime 2 } + a _ { 3 3 } ^ { \prime } x _ { 3 } ^ { \prime 2 } = 0 } \\
{ x _ { 3 } ^ { \prime } = 0 }
\end{array} \text { and } \left\{\begin{array}{l}
b_{11}^{\prime} x_{1}^{\prime 2}+b_{22}^{\prime} x_{2}^{\prime 2}+b_{33}^{\prime} x_{3}^{\prime 2}=0 \\
x_{3}^{\prime}=0
\end{array}\right.\right.
$$

If we denote the projective coordinates of $M_{1}, N_{1}, M_{2}$ and $N_{2}$ on $X Y$ as $\theta_{1}, \theta_{1}^{\prime}, \theta_{2}$ and $\theta_{2}^{\prime}$, they are respectively the roots of

$$
\left(\frac{x_{2}^{\prime}}{x_{1}^{\prime}}\right)^{2}=-\frac{a_{11}^{\prime}}{a_{22}^{\prime}} \text { and }\left(\frac{x_{2}^{\prime}}{x_{1}^{\prime}}\right)^{2}=-\frac{b_{11}^{\prime}}{b_{22}^{\prime}} .
$$

As homogeneous coordinates are unchanged by an arbitrary scalar, so only ratios of homogeneous coordinates are significant. Let consider one of the ratios $\lambda_{i} / \lambda_{j}$, say $\lambda_{1} / \lambda_{2}$.

Obviously we have $\frac{\lambda_{1}}{\lambda_{2}}=\frac{a_{j 2}^{\prime} / b_{11}^{\prime}}{a_{22}^{\prime} / b_{22}^{\prime}}$. On the other hand, the cross-ratio of $M_{1}, M_{2}$ with respect to $X, Y$ is $\left\{M_{1}, M_{2} ; X, Y\right\}=\left\{\theta_{1}, \theta_{2} ; \infty, 0\right\}=\frac{\theta_{2}}{\theta_{1}}$.

It comes

$$
\left\{M_{1}, M_{2} ; X, Y\right\}^{2}=\left(\frac{\theta_{2}}{\theta_{1}}\right)^{2}=\frac{a_{11}^{\prime} / a_{22}^{\prime}}{b_{11}^{\prime} / b_{22}^{\prime}} .
$$

So $\lambda_{1} / \lambda_{2}=\left\{M_{1}, M_{2} ; X, Y\right\}^{2}$. 
Note that

$$
\left\{M_{1}, M_{2} ; X, Y\right\}^{2}=\left\{M_{1}, N_{2} ; X, Y\right\}^{2}=\left\{N_{1}, M_{2} ; X, Y\right\}^{2}=\left\{N_{1}, N_{2} ; X, Y\right\}^{2} .
$$

That means that the ratio of two eigenvalues is the square of the cross-ratio of $M_{1}, M_{2}$ (either $M_{1}, N_{2}$ or $N_{1}, M_{2}$ or $N_{1}, N_{2}$ ) with respect to $X$ and $Y$. Similarly, other ratios of eigenvalues have the same geometric interpretation in terms of cross ratios.

\subsection{Invariants of a pair of conics and their interpretation}

Now it is the turn to give a geometric interpretation of invariants of conics presented at the begining.

Taking the reduced form of the conics through the common self-polar triangle, we have

$$
\begin{array}{ll}
I_{1}=a_{11}^{\prime} a_{22}^{\prime} a_{33}^{\prime}, & I_{3}=a_{11}^{\prime} B_{11}^{\prime}+a_{22}^{\prime} B_{22}^{\prime}+a_{33}^{\prime} B_{33}^{\prime}, \\
I_{2}=b_{11}^{\prime} A_{11}^{\prime}+b_{22}^{\prime} A_{22}^{\prime}+b_{33}^{\prime} A_{33}^{\prime}, & I_{4}=b_{11}^{\prime} b_{22}^{\prime} b_{33}^{\prime} .
\end{array}
$$

Three invariants of the associated quadratic forms are

$$
\frac{I_{3}}{I_{4}}=\lambda_{1}+\lambda_{2}+\lambda_{3}, \frac{I_{2}}{I_{1}}=\frac{1}{\lambda_{1}}+\frac{1}{\lambda_{2}}+\frac{1}{\lambda_{3}} \text { and } \frac{I_{1}}{I_{4}}=\lambda_{1} \lambda_{2} \lambda_{3} .
$$

All other ratios $I_{i} / I_{j}$, for $i \neq j$ (invariants of quadratic forms) can be expressed from these three and are symmetric functions of $\lambda_{1}, \lambda_{2}, \lambda_{3}$.

Obviously,

$$
\operatorname{Trace}\left(\mathbf{C}_{2}^{-1} \mathbf{C}_{1}\right)=\frac{I_{3}}{I_{4}}, \operatorname{Trace}\left(\mathbf{C}_{1}^{-1} \mathbf{C}_{2}\right)=\frac{I_{2}}{I_{1}} \text { and } \frac{\left|\mathbf{C}_{1}\right|}{\left|\mathbf{C}_{2}\right|}=\frac{I_{1}}{I_{4}},
$$

so that Trace $\left(\mathbf{C}_{2}^{-1} \mathbf{C}_{1}\right), \operatorname{Trace}\left(\mathbf{C}_{1}^{-1} \mathbf{C}_{2}\right)$ and $\left|\mathbf{C}_{1}\right| /\left|\mathbf{C}_{2}\right|$ are only invariants of a pair of quadratic forms, but not invariants of the conics. We can not express them in terms of ratios of $\lambda_{i}$ which are some known cross ratios.

Two of true invariants of the conics are

$$
\alpha=\frac{I_{1} I_{3}}{I_{2}^{2}}=\frac{\operatorname{Trace}\left(\mathbf{C}_{2}^{-1} \mathbf{C}_{1}\right)}{\left(\operatorname{Trace}\left(\mathbf{C}_{1}^{-1} \mathbf{C}_{2}\right)\right)^{2}} \frac{\left|\mathbf{C}_{2}\right|}{\left|\mathbf{C}_{1}\right|}, \quad \beta=\frac{I_{2} I_{4}}{I_{3}^{2}}=\frac{\operatorname{Trace}\left(\mathbf{C}_{1}^{-1} \mathbf{C}_{2}\right)}{\left(\operatorname{Trace}\left(\mathbf{C}_{2}^{-1} \mathbf{C}_{1}\right)\right)^{2}} \frac{\left|\mathbf{C}_{1}\right|}{\left|\mathbf{C}_{2}\right|} .
$$

They should be expressed in terms of some known cross ratios. It can be done easily:

$$
\begin{aligned}
& \alpha=\frac{I_{1} I_{3}}{I_{2}^{2}}=\frac{\left(\lambda_{1}+\lambda_{2}+\lambda_{3}\right)\left(\lambda_{1} \lambda_{2} \lambda_{3}\right)}{\left(\lambda_{1} \lambda_{2}+\lambda_{2} \lambda_{3}+\lambda_{1} \lambda_{3}\right)^{2}}=\lambda_{3} / \lambda_{2} \frac{1+\lambda_{2} / \lambda_{1}+\lambda_{3} / \lambda_{1}}{\left(1+\lambda_{3} / \lambda_{1}+\lambda_{3} / \lambda_{2}\right)^{2}}, \\
& \beta=\frac{I_{2} I_{4}}{I_{3}^{2}}=\frac{\lambda_{1} \lambda_{2}+\lambda_{2} \lambda_{3}+\lambda_{1} \lambda_{3}}{\left(\lambda_{1}+\lambda_{2}+\lambda_{3}\right)^{2}}=\lambda_{2} / \lambda_{1} \frac{1+\lambda_{3} / \lambda_{1}+\lambda_{3} / \lambda_{2}}{\left(1+\lambda_{2} / \lambda_{1}+\lambda_{3} / \lambda_{1}\right)^{2}} .
\end{aligned}
$$

Every ratios $\lambda_{i} / \lambda_{j}$ are the squares of some known cross ratios, so that the invariants of a pair of conics are rational functions of some known cross ratios.

Note that Forsyth et al. $[1,2]$ propose $\operatorname{Trace}\left(\mathbf{C}_{2}^{-1} \mathbf{C}_{1}\right)$ and $\operatorname{Trace}\left(\mathbf{C}_{1}^{-1} \mathbf{C}_{2}\right)$ as the two invariants of a pair of conics. They are actually only invariants for 
the associated quadratics, not for the conics. They become the true invariants of conics when the associated matrices are normalized. As normalization makes no scaling possible, a quadratic form is uniquely associated with a conic. On the other hand, with $\left|\mathbf{C}_{1}\right|=I_{1}=1\left|\mathbf{C}_{2}\right|=I_{4}=1, \operatorname{Trace}\left(\mathbf{C}_{2}^{-1} \mathbf{C}_{1}\right)$ and $\operatorname{Trace}\left(\mathbf{C}_{1}^{-1} \mathbf{C}_{2}\right)$ can be expressed in rational functions of $\alpha$ and $\beta$ :

$$
\operatorname{Trace}\left(\mathbf{C}_{2}^{-1} \mathbf{C}_{1}\right)=\left(\frac{1}{\alpha^{2} \beta}\right)^{\frac{2}{3}} \text { and } \operatorname{Trace}\left(\mathbf{C}_{1}^{-1} \mathbf{C}_{2}\right)=\left(\frac{1}{\alpha \beta^{2}}\right)^{\frac{2}{3}} \text {. }
$$

\section{Conclusion}

The invariants of a pair of conics are first revisited and then they are geometrically interpreted based on cross ratios with the help of the common self-polar triangle of the conics. This work is a continuation of the invariant investigation of Forsyth et al. We hope to provide a better comprehension and use of conics invariants in computer vision. Our conviction is that cross ratio is the simplest fundamental projective invariant. Other invariants, at least those concerning point, line and conic entities, should be expressible by cross ratios. Therefore, the invariants for a set of geometric entities can often be directly constructed. For example, to get the two invariants of a pair of conics, the most direct ones are just the two cross ratios of the four intersection points defined for each conic [4]. However, the stability of invariants such way obtained with respect to the other invariants should be verified through experimentation.

Acknowledgements We would like to thank Prof. Michel Brion and Oscar Burlet for many interesting discussions.

\section{References}

[1] D. Forsyth, J. Mundy, and A. Zisserman. Transformationnal Invariance - a primer. In British Machine Vision Conference, pages 1-6, Sept. 1990.

[2] D. Forsyth, J. L. Mundy, A. Zisserman, and C. Rothwell. Invariant Descriptors for 3D Object Recognition and Pose. In Applications of Invariance in Computer Vision, pages 171 - 208, Reykjavik - Iceland, 25 - 28 March 1991. DARPA - ESPRIT Workshop.

[3] D. Kapur and J. L. Mundy. Fitting Affine Invariant Conics to Curves. In Applications of Invariance in Computer Vision, pages 209 - 233, Reykjavik - Iceland, 25 - 28 March 1991. DARPA - ESPRIT Workshop.

[4] S.J. Maybank. The projection of two non-coplanar conics. In Applications of Invariance in Computer Vision, pages pages 47 - 53, Reykjavik - Iceland, 25 - 28 March 1991. DARPA - EEC Workshop.

[5] J.G. Semple and G.T. Kneebone. Algebraic Projective Geometry. Oxford Science Publication, 1952.

[6] A. Zisserman, C. Marinos, D.A. Forsyth, J.L. Mundy, and C.A. Rothwell. Relative Motion and Pose from Invariants. In British Machine Vision Conference, pages 7-12, 1990. 